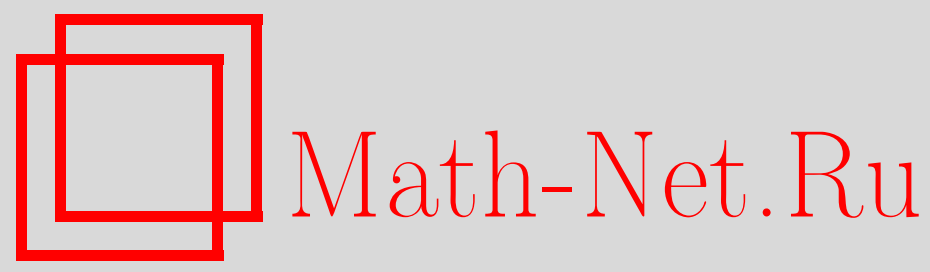

В. Н. Коновалов, О порядках нелинейных приближений классов функций заданной формы, Матем. заметки, 2005, том 78, выпуск 1, 98-114

DOI: https://doi.org/10.4213/mzm2565

Использование Общероссийского математического портала Math-Net.Ru подразумевает, что вы прочитали и согласны с пользовательским соглашением http://www . mathnet.ru/rus/agreement

Параметры загрузки:

IP: 54.162 .85 .209

26 апреля 2023 г., 15:35:09

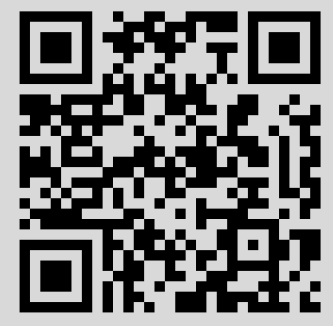




\section{О ПОРЯДКАХ НЕЛИНЕЙНЫХ ПРИБЛИЖКЕИЙ КЛАССОВ ФУНКЦИЙ ЗАДАННОЙ ФОРМЫ}

\section{В.Н. Коновалов}

Пусть $\Delta_{+}^{s}-$ множество функций $x: I \rightarrow \mathbb{R}$ на конечном интервале $I$ таких, что разделенные разности $\left[x ; t_{0}, \ldots, t_{s}\right]$ порядка $s \in \mathbb{N}$ этих функций являются неотрицательными для всех наборов из $(s+1)$ различных точек $t_{0}, \ldots, t_{s} \in I$. При всех $s \in \mathbb{N}$ и $1 \leqslant p \leqslant \infty$ установлены точные порядки наилучших приближений сплайнами со свободными узлами и рациональными функциями в метриках $L_{p}$ классов $\Delta_{+}^{s} B_{p}:=$ $\Delta_{+}^{s} \cap B_{p}$, где $B_{p}$ - единичный шар в $L_{p}$. Также установлена асимптотика псевдо-размерностных поперечников в $L_{p}$ этих классов функций.

Библиографоия: 13 названий.

1. Введение. Формулировки основных результатов. При $s \in \mathbb{N}$ функцию $x: I \rightarrow \mathbb{R}$ будем назьвать $s$-монотонной на промежутке $I \subseteq \mathbb{R}$ (интервале, полуинтервале или отрезке), если для всех наборов из $(s+1)$ различных точек $t_{0}, \ldots, t_{s} \in I$ соответствующие разделенные разности $\left[x ; t_{0}, \ldots, t_{s}\right]$ порядка $s$ этой функции являются неотрицательными. Очевидно, что $s$-монотонные функции при $s=1$ и $s=2$ это, соответственно, неубьвающие и вьпуклые функции на $I$. Следовательно, параметр $s$ характеризует форму функций. По поводу свойств $s$-монотонных функций смотри [1]- [3].

Класс всех $s$-монотонных на $I$ функций будем обозначать через $\Delta_{+}^{s}(I)$. Кроме того, если на $I$ определен некоторьй класс функций $W(I)$, то полагаем $\Delta_{+}^{s} W(I):=\Delta_{+}^{s}(I) \cap$ $W(I)$. Через $L_{p}(I), 1 \leqslant p \leqslant \infty$, обозначаем, как обычно, линейное пространство всех измеримых по Лебегу функций $x: I \rightarrow \mathbb{R}$ с конечной нормой $\|x\|_{L_{p}(I)}$. При $p=\infty$ под $\|x\|_{L_{\infty}(I)}$ понимается ess sup- или sup-норма. Единичньй шар пространства $L_{p}(I)$ обозначаем через $B_{p}(I)$. Для промежутка $I$ с концами в точках $a:=-1$ и $b:=1$ его обозначение будет иногда опускаться. Например, если $I=(-1,1)$, то $W:=W(I)$.

Обозначим через $P_{n}(I)$, где $n \in \mathbb{N}$, множество всех алгебраических многочленов $\pi_{n}$ порядка $\leqslant n$ (т.е. степени $\leqslant n-1$ ), определенных на промежутке $I$. Через $R_{n}(I)$ обозначаем множество всех рациональных функций на $I$, имеющих вид $\rho_{n}=\hat{\pi}_{n} / \check{\pi}_{n}$, где $\hat{\pi}_{n}, \check{\pi}_{n} \in P_{n}$. При этом предполагается, что $\check{\pi}_{n} \not \equiv 0$. В тех точках $t \in I$, где $\check{\pi}_{n}(t)=0$, функция $\rho_{n}$ может принимать произвольные конечные значения. Если на промежутке $I$ с концами в точках $a:=-1$ и $b:=1$ задан некоторьй класс $W$ функций $x$ такой, что $W \subset L_{q}$, то через

$$
E\left(W, R_{n}\right)_{L_{q}}:=\sup _{x \in W} \inf _{\rho_{n} \in R_{n}}\left\|x-\rho_{n}\right\|_{L_{q}}
$$

обозначим величину его наилучшего приближения в $L_{q}$ множеством $R_{n}$. 
А.П. Булановьм [4] было установлено, что для множества $\Delta_{+}^{2} B_{\infty}$ всех вьпуклых на интервале $I:=(-1,1)$ функций $x$, удовлетворяющих ограничению $\|x\|_{L_{\infty}} \leqslant 1$, справедливы двухсторонние оценки

$$
c_{1} n^{-1} \leqslant E\left(\Delta_{+}^{2} B_{\infty}, R_{n}\right)_{L_{\infty}} \leqslant c_{2} n^{-1} \log ^{2}(n+1), \quad n \geqslant 1
$$

где $c_{1}>0$ и $c_{2}>0-$ абсолютные постоянные.

Затем В.А. Попов и П. П. Петрушев [5] усилили оценку сверху, доказав, что

$$
E\left(\Delta_{+}^{2} B_{\infty}, R_{n}\right)_{L_{\infty}} \leqslant c n^{-1}, \quad n \geqslant 1
$$

где $c>0$ - абсолютная постоянная. Таким образом, был найден точньй порядок

$$
c_{1} n^{-1} \leqslant E\left(\Delta_{+}^{2} B_{\infty}, R_{n}\right)_{L_{\infty}} \leqslant c_{2} n^{-1}, \quad n \geqslant 1,
$$

наилучших приближений в $L_{\infty}$ класса $\Delta_{+}^{2} B_{\infty}$ рациональньми функциями.

В связи с этим результатом возникает ряд вопросов. Например, можно ли получить подобную оценку для наилучших приближений классов $\Delta_{+}^{2} B_{p}$ в метриках $L_{p}$ при $1 \leqslant p<\infty$ ? Каковы порядки наилучших приближений рациональными функциями в $L_{p}$ при $1 \leqslant p \leqslant \infty$ классов $\Delta_{+}^{s} B_{p}$ для $s \neq 2$ ? Могут ли сплайны обеспечить приближения по порядку лучшие, чем рациональные функции? Реализуют ли множества рациональных функций и сплайнов наилучший порядок приближения, по сравнению с другими нелинейными многообразиями функций, имеющими заданную “размерность”?

В данной работе предлагаются ответы на эти вопросы. Точнее, решается задача о порядковой асимптотике наилучших приближений классов $\Delta_{+}^{s} B_{p}$ при всех $s \in \mathbb{N}$ в метриках $L_{p}, 1 \leqslant p \leqslant \infty$, сплайнами со свободными узлами и рациональньми функциями. Кроме того, для этих классов устанавливаются точные порядки псевдо-размерностных поперечников.

Через $\Sigma_{r, n}(I)$, где $r, n \in \mathbb{N}$, обозначим множество всех определенных на промежутке $I$ кусочно-полиномиальных сплайнов $\sigma_{r, n}$ порядка $\leqslant r$ с не более чем $(n-1)$ свободными узлами. По определению $\sigma_{r, n} \in \Sigma_{r, n}(I)$, если существует некоторый набор $\left\{I_{i}\right\}_{i=1}^{m}$ из $m \leqslant n$ попарно непересекающихся интервалов $I_{i} \subseteq I$, суммарная длина которых равна длине $I$, и таких, что на каждом из интервалов $I_{i}$ сплайн $\sigma_{r, n}$ совпадает с некоторым многочленом $\pi_{r}\left(\cdot ; I_{i}\right) \in P_{r}\left(I_{i}\right)$. Точки промежутка $I$, в которых сплайн $\sigma_{r, n}$ или хотя бы одна из его производных имеют разрьв, называются узлами. Узлы, лежащие внутри промежутка $I$, будем назьвать свободными.

Псевдо-размерность совокупности $M:=\{x\} \neq \varnothing$ функций $x: T \rightarrow \mathbb{R}$, заданных на произвольном множестве $T \neq \varnothing$, определяется следующим образом (см. [6, раздел 4 , определение 3]). Для чисел $a \in \mathbb{R}$ полагаем $\operatorname{sgn} a:=1$ при $a>0$ и $\operatorname{sgn} a:=0$ при $a \leqslant 0$. Псевдо-размерностью $\operatorname{dim}_{p s}(M)$ множества функций $M$ назьвается наибольшее из чисел $n \in \mathbb{N}$ таких, что для каждого из них существуют набор точек $t_{1}, \ldots, t_{n} \in T$ и вектор $a:=\left(a_{1}, \ldots, a_{n}\right) \in \mathbb{R}^{n}$ такие, что

$$
\operatorname{card}\left\{\left(\operatorname{sgn}\left(x\left(t_{1}\right)+a_{1}\right), \ldots, \operatorname{sgn}\left(x\left(t_{n}\right)+a_{n}\right)\right) \mid x \in M\right\}=2^{n} .
$$

Если конечного $n$ не существует, то $\operatorname{dim}_{p s}(M):=\infty$, а если $M$ состоит лиш из одной функции, то $\operatorname{dim}_{p s}(M):=0$. 
Псевдо-размерность обобщает понятие обычной размерности $\operatorname{dim}(M)$ линейных пространств $M$ действительно-значных функций, так как известно (см. [6, раздел 4, теорема 4]), что в этом случае $\operatorname{dim}(M)=\operatorname{dim}_{p s}(M)$.

Известно также, что для множеств $\Sigma_{r, n}=\Sigma_{r, n}(I)$ сплайнов и множеств $R_{n}=R_{n}(I)$ рациональных функций имеют место соотношения

$$
n \leqslant \operatorname{dim}_{p s}\left(\Sigma_{r, n}\right) \leqslant c n, \quad n \leqslant \operatorname{dim}_{p s}\left(R_{n}\right) \leqslant c n, \quad n \geqslant 1,
$$

где $c>0$ не зависит от $n$. Оценки снизу в этих соотношениях очевидны, так как множество $\Sigma_{r, n}$ содержит пространство сплайнов (с фиксированньми узлами) размерности $n$, а множество $R_{n}$ содержит $n$-мерное пространство $P_{n}$ многочленов порядка $n$. Оценки сверху нетрудно получить непосредственно из определения псевдо-размерности, если учесть, что сплайны из $\Sigma_{r, n}(I)$ и рациональные функции из $R_{n}(I)$ могут менять знак на промежутке $I$ не более, чем $c n$ раз, где $c$ не зависит от $n$.

Д. Ратсаби и В. Майоров [7], [8], используя понятие псевдо-размерности, ввели новые нелинейные поперечники, определив их следующим образом. Пусть $X=X(T)-$ линейное пространство функций $x: T \rightarrow \mathbb{R}$ с нормой $\|x\|_{X}$, a $W=W(T)$ - произвольное непустое подмножество в $X$. Тогда величина

$$
d_{n}(W)_{X}^{p s d}:=\inf _{M^{n}} \sup _{x \in W} \inf _{y \in M^{n}}\|x-y\|_{X}
$$

где первый инфимум взят по всем $M^{n} \subseteq X$ псевдо-размерности $\leqslant n$, назьвается $n c e \boldsymbol{\text { - }}$ до-размерностным $n$-поперечником множества $W$ в $X$.

Прежде чем сформулировать полученные результаты, условимся еше о некоторых обозначениях. Через $|I|$ будут обозначаться длины промежутков $I$, а через $c:=c(\alpha, \beta$, $\ldots, \gamma)$ - различные положительные "постоянные", зависящие от параметров $\alpha, \beta, \ldots, \gamma$. Если заданы две последовательности $\left\{a_{n}\right\}$ и $\left\{b_{n}\right\}$ положительных чисел $a_{n}$ и $b_{n}$, то эти последовательности удовлетворяют соотношению $a_{n} \asymp b_{n}, n \geqslant 1$, тогда и только тогда, когда существуют, не зависящие от $n$, постоянные $c_{1}>0$ и $c_{2}>0$ такие, что $c_{1} \leqslant a_{n} / b_{n} \leqslant c_{2}$ для всех $n \geqslant 1$.

Основные результатыприведены в следующей теореме, гдепредполагается, что классы $\Delta_{+}^{s} B_{p}$ заданы на интервале $I:=(-1,1)$. В формулировку теоремы включен и результат о наилучшем равномерном приближении класса $\Delta_{+}^{2} B_{\infty}$ рациональными функциями, принадлежащий А. П. Буланову [4], В. А. Попову и П. П. Петрушеву [5].

Tеорема 1. Ecлu $r, s \in \mathbb{N}, s>1 u p=\infty, m o$

$$
d_{n}\left(\Delta_{+}^{s} B_{\infty}\right)_{L_{\infty}}^{p s d} \asymp E\left(\Delta_{+}^{s} B_{\infty}, \Sigma_{r, n}\right)_{L_{\infty}} \asymp E\left(\Delta_{+}^{s} B_{\infty}, R_{n}\right)_{L_{\infty}} \asymp n^{-1}, \quad n \geqslant 1 .
$$

Ecлu $r \in \mathbb{N}, s=1 u p=\infty, m o$

$$
d_{n}\left(\Delta_{+}^{1} B_{\infty}\right)_{L_{\infty}}^{p s d} \asymp E\left(\Delta_{+}^{1} B_{\infty}, \Sigma_{r, n}\right)_{L_{\infty}} \asymp n^{-1}, \quad n \geqslant 1,
$$

но при этом

$$
E\left(\Delta_{+}^{1} B_{\infty}, R_{n}\right)_{L_{\infty}} \asymp 1, \quad n \geqslant 1 .
$$

Eсли $r, s \in \mathbb{N} u 1 \leqslant p<\infty$, mo

$$
d_{n}\left(\Delta_{+}^{s} B_{p}\right)_{L_{p}}^{p s d} \asymp E\left(\Delta_{+}^{s} B_{p}, \Sigma_{r, n}\right)_{L_{p}} \asymp E\left(\Delta_{+}^{s} B_{p}, R_{n}\right)_{L_{p}} \asymp 1, \quad n \geqslant 1 .
$$


2. Вспомогательные утверждения. При доказательстве оценок снизу в теореме 1 будут использоваться следуюшие свойства псевдо-размерности.

Лемма 2. Пусть на множестве $T \neq \varnothing$ задано семейство $X=X(T)$ функиий $x: T \rightarrow \mathbb{R}$. Тогда

1) если $y-$ фиксированная функиия, $а y+X:=\{z \mid z=y+x, x \in X\}$, то $\operatorname{dim}_{p s}(y+X)=\operatorname{dim}_{p s}(X)$

2) если $y(t)>0$ при всех $t \in T$, a $y \cdot X:=\{z \mid z=y \cdot x, x \in X\}$, mo $\operatorname{dim}_{p s}(y \cdot X)=\operatorname{dim}_{p s}(X)$

3) если отобрахение $\theta: T \rightarrow T$ является взаимно-однозначным, $a$

$$
X_{(\theta)}:=\left\{x_{(\theta)} \mid x_{(\theta)}(t):=x(\theta(t)), t \in T, x \in X\right\},
$$

$m o \operatorname{dim}_{p s}\left(X_{(\theta)}\right)=\operatorname{dim}_{p s}(X) ;$

4) если $T_{*} \subseteq T, a X_{\mid T_{*}}:=\left\{x_{\mid T_{*}} \mid x_{\mid T_{*}}(t):=x(t), t \in T_{*}, x \in X\right\}$, mo $\operatorname{dim}_{p s}\left(X_{\mid T_{*}}\right)$ $\leqslant \operatorname{dim}_{p s}(X)$

5) если $a>0, a X_{a}:=\left\{x_{a}\right\}$ - множсество функиий-срезок $x_{a}$ таких, что $x_{a}(t):=x(t)$ в случае $|x(t)| \leqslant a u x_{a}(t):=a \operatorname{sgn} x(t)$ в случае $|x(t)|>a$, то $\operatorname{dim}_{p s}\left(X_{a}\right) \leqslant \operatorname{dim}_{p s}(X)$.

По поводу свойства 1) см. [6, раздел 4, теорема 5], а свойства 2) - 5) легко выводятся непосредственно из определения псевдо-размерности.

Если $m \in \mathbb{N}$, то через $l_{1}^{m}$ обозначаем, как обычно, пространство векторов $x:=\left(x_{1}\right.$, $\left.\ldots, x_{m}\right) \in \mathbb{R}^{m}$ с нормой $\|x\|_{l_{1}^{m}}:=\sum_{i=1}^{m}\left|x_{i}\right|$.

Приведенная ниже лемма 3 является немедленньм следствием из [9, гл. 15, $\S 2$, лемма 2.2]. Доказательство леммы 3 дано также в [10, раздел 4 , лемма 1].

Лемма 3. Пусть $\{-1,1\}^{m}:=\left\{v \mid v:=\left(v_{1}, \ldots, v_{m}\right), v_{i}= \pm 1, i=1, \ldots, m\right\}$, где $m \in \mathbb{N}$. Тогда существует подмножество $V^{(m)} \subset\{-1,1\}^{m}$ такое, что $\operatorname{card}\left(V^{(m)}\right) \geqslant 2^{m / 16}$, и при этом

$$
\|\hat{v}-\check{v}\|_{l_{1}^{m}} \geqslant \frac{m}{2}, \quad \hat{v}, \check{v} \in V^{(m)}, \quad \hat{v} \neq \check{v} .
$$

Пусть $X$ - линейное пространство элементов $x$ с нормой $\|x\|_{X}$. При заданном $\varepsilon>0$ точки $\hat{x}$ и $\check{x}$ назьваются $\varepsilon$-различимыми по норме $X$, если $\|\hat{x}-\check{x}\|_{X} \geqslant \varepsilon$. Для каждого подмножества $M \neq \varnothing$ из $X$ обозначим через $\mathscr{N}_{\varepsilon}(M)$ максимальное натуральное число $\varepsilon$-различимых по норме $X$ точек $\mu \in M$, если такое число существует. А если конечного $\mathscr{N}_{\varepsilon}(M)$ не существует, то полагаем $\mathscr{N}_{\varepsilon}(M):=\infty$.

Следующая лемма, является немедленным следствием из [11, раздел 3.3, следствие 3$]$. Эта лемма приведена в [12, раздел 3, лемма 1$]$.

Лемма 4. Пусть $I=(0,1), n \in \mathbb{N}, a>0, a M_{a}^{n}:=M_{a}^{n}(I)$-множество функиий $\mu: I \rightarrow \mathbb{R}$ из $L_{\infty}(I)$ таких, что $\|\mu\|_{L_{\infty}(I)} \leqslant$ a $u \operatorname{dim}_{p s}\left(M_{a}^{n}\right) \leqslant n$. Тогда при кажсдом $\varepsilon>0$ имеет место оценка

$$
\mathscr{N}_{\varepsilon}\left(M_{a}^{n}\right)_{L_{1}(I)} \leqslant e(n+1)\left(\frac{4 e a}{\varepsilon}\right)^{n} .
$$

Доказательство следующей леммы базируется на рассуждениях, которые применялись для получения оценок снизу в [12, теорема 1$]$. 
ЛЕмма 5. Пусть $I:=(0,1)$, а числа $m \in \mathbb{N}, a>0$ и $\alpha>0$ удовлетворяют неравенству $m \geqslant 16\left(8+\log _{2}(a / \alpha)\right)$. Пусть $\Phi^{(m)}=\Phi^{(m)}(I)-$ множсество функиий $\varphi: I \rightarrow \mathbb{R}$ из $L_{\infty}(I)$ такое, ито $\operatorname{card}\left(\Phi^{(m)}\right) \geqslant 2^{m / 16},\|\varphi\|_{L_{\infty}(I)} \leqslant a, u$

$$
\|\hat{\varphi}-\check{\varphi}\|_{L_{1}(I)} \geqslant \alpha, \quad \hat{\varphi}, \check{\varphi} \in \Phi^{(m)}, \quad \hat{\varphi} \neq \check{\varphi} .
$$

Тогда при каждом $n \in \mathbb{N}$ таком, ито $n \leqslant\left(16\left(8+\log _{2}(a / \alpha)\right)\right)^{-1} m$, будет справедлива оченка

$$
d_{n}\left(\Phi^{(m)}\right)_{L_{1}(I)}^{p s d} \geqslant \frac{\alpha}{8}
$$

ДокАЗАтЕльство. Зафиксировав натуральные числа $m \geqslant 16\left(8+\log _{2}(a / \alpha)\right)$ и $n \leqslant$ $\left(16\left(8+\log _{2}(a / \alpha)\right)\right)^{-1} m$, зафиксируем также произвольное множество $M^{n}:=M^{n}(I)$ из $L_{1}(I)$ такое, что $\operatorname{dim}_{p s}\left(M^{n}\right) \leqslant n$. Полагаем

$$
\delta:=E\left(\Phi^{(m)}, M^{n}\right)_{L_{1}(I)}:=\sup _{\varphi \in \Phi^{(m)}} \inf _{\mu \in M^{n}}\|\varphi-\mu\|_{L_{1}(I)}
$$

Сопоставляя каждой функции $\varphi \in \Phi^{(m)}$ функцию $\mu_{\delta}(\cdot ; \varphi) \in M^{n}$ такую, что

$$
\left\|\varphi(\cdot)-\mu_{\delta}(\cdot ; \varphi)\right\|_{L_{1}(I)} \leqslant 2 \delta
$$

полагаем $M_{\delta}^{n}:=M_{\delta}^{n}(I):=\left\{\mu_{\delta}(\cdot ; \varphi), \varphi \in \Phi^{(m)}\right\}$. Очевидно, что $M_{\delta}^{n} \subseteq M^{n}$.

Полагая $\mu_{\delta, a}(t ; \varphi):=\mu_{\delta}(t ; \varphi)$ в случае $\left|\mu_{\delta}(t ; \varphi)\right| \leqslant a$ и $\mu_{\delta, a}(t ; \varphi):=a \operatorname{sgn} \mu_{\delta}(t ; \varphi)$ в случае $\left|\mu_{\delta}(t ; \varphi)\right|>a$, обозначим через $M_{\delta, a}^{n}:=\left\{\mu_{\delta, a}(\cdot ; \varphi), \varphi \in \Phi^{(m)}\right\}$ множество соответствующих функций-срезок. Очевидно, что

$$
\left\|\mu_{\delta, a}(\cdot ; \varphi)\right\|_{L_{\infty}(I)} \leqslant a, \quad \varphi \in \Phi^{(m)} .
$$

Так же ясно, что из включения $M_{\delta}^{n} \subseteq M^{n}$ и утверждения 4) леммы 2 следуют неравенства

$$
\operatorname{dim}_{p s}\left(M_{\delta, a}^{n}\right) \leqslant \operatorname{dim}_{p s}\left(M_{\delta}^{n}\right) \leqslant \operatorname{dim}_{p s}\left(M^{n}\right) \leqslant n .
$$

Докажем теперь, что $\delta>\alpha / 8$. Допуская от противного, что $\delta \leqslant \alpha / 8$, и зафиксировав произвольные функции $\hat{\varphi}, \check{\varphi} \in \Phi^{(m)}$ такие, что $\hat{\varphi} \neq \check{\varphi}$, имеем

$$
\begin{gathered}
\left\|\mu_{\delta, a}(\cdot ; \hat{\varphi})-\mu_{\delta, a}(\cdot ; \check{\varphi})\right\|_{L_{1}(I)} \geqslant \\
\|\hat{\varphi}-\check{\varphi}\|_{L_{1}(I)}-\left\|\hat{\varphi}(\cdot)-\mu_{\delta, a}(\cdot ; \hat{\varphi})\right\|_{L_{1}(I)} \\
-\left\|\check{\varphi}(\cdot)-\mu_{\delta, a}(\cdot ; \check{\varphi})\right\|_{L_{1}(I)} .
\end{gathered}
$$

А так как нормы $\hat{\varphi}$ и $\check{\varphi}$ в $L_{\infty}(I)$ не превосходят $a$, то

$$
\begin{aligned}
& \left\|\hat{\varphi}(\cdot)-\mu_{\delta, a}(\cdot ; \hat{\varphi})\right\|_{L_{1}(I)} \leqslant\left\|\hat{\varphi}(\cdot)-\mu_{\delta}(\cdot ; \hat{\varphi})\right\|_{L_{1}(I)} \\
& \left\|\check{\varphi}(\cdot)-\mu_{\delta, a}(\cdot ; \check{\varphi})\right\|_{L_{1}(I)} \leqslant\left\|\check{\varphi}(\cdot)-\mu_{\delta}(\cdot ; \check{\varphi})\right\|_{L_{1}(I)}
\end{aligned}
$$

Из этих неравенств, а также из (2.2) и (2.5) следует, что

$$
\left.\| \mu_{\delta, a}(\cdot ; \hat{\varphi})-\mu_{\delta, a}(\cdot ; \check{\varphi})\right)\left\|_{L_{1}(I)} \geqslant\right\| \hat{\varphi}-\check{\varphi} \|_{L_{1}(I)}-4 \delta \geqslant \frac{\alpha}{2} .
$$


Если положить $\varepsilon:=\alpha / 2$, то из (2.6) следует, что множество $M_{\delta, a}^{n}$ состоит из $\varepsilon$-различимых по норме $L_{1}(I)$ функций. Кроме того, для этих функций вьполняются неравенства (2.3). Учитывая также (2.4) и воспользовавшись леммой 4 , получаем

$$
\mathscr{N}_{\alpha / 2}\left(M_{\delta, a}^{n}\right)_{L_{1}(I)} \leqslant e(n+1)\left(\frac{8 e a}{\alpha}\right)^{n}<2^{3 n}\left(\frac{2^{5} a}{\alpha}\right)^{n}=2^{\left(8+\log _{2}(a / \alpha)\right) n} .
$$

А так как $\operatorname{card}\left(M_{\delta, a}^{n}\right) \geqslant 2^{m / 16}$, где $m \geqslant 16\left(8+\log _{2}(a / \alpha)\right) n$, мы приходим к неравенствам

$$
2^{\left(8+\log _{2}(a / \alpha)\right) n} \leqslant \mathscr{N}_{\alpha / 2}\left(M_{\delta, a}^{n}\right)_{L_{1}(I)}<2^{\left(8+\log _{2}(a / \alpha)\right) n}
$$

которые не могут выполняться одновременно. Полученное противоречие доказывает справедливость неравенства $\delta>\alpha / 8$, где $\delta$ определено согласно (2.1).

Но тогда в силу произвольности выбора множества $M^{n}$ получаем

$$
d_{n}\left(\Phi^{(m)}\right)_{L_{1}(I)} \geqslant \frac{\alpha}{8}
$$

Лемма 5 доказана.

Приведенная ниже лемма 6 доказана в [13, лемма 3]. В этой лемме и далее для функций $x: I \rightarrow \mathbb{R}$, имеющих в точке $t \in I$ интервала $I$ конечные односторонние производные $x_{-}^{(k)}(t)$ и $x_{+}^{(k)}(t)$ порядка $k \in \mathbb{N}$, полагаем

$$
x^{(k)}(t):=\frac{1}{2}\left(x_{-}^{(k)}(t)+x_{+}^{(k)}(t)\right) .
$$

Ясно, что если в какой-либо точке интервала $I$ существует обычная производная порядка $k \in \mathbb{N}$, то она совпадает с так определяемой обобщенной производной. При $k=0$ полагаем $x^{(0)}(t):=x(t), t \in I$. Отметим также, что если $x \in \Delta_{+}^{s}(I)$, где $s>1$, то в каждой точке $t \in I$ существуют при всех $k=1, \ldots, s-1$ односторонние производные $x_{-}^{(k)}(t)$ и $x_{+}^{(k)}(t)$. При этом на $I$ существует обычная производная $x^{(s-2)}$, являющаяся выпуклой и локально абсолютно непрерывной на $I$ функцией. Ясно, что обобщенная производная $x^{(s-1)}$ является неубывающей на $I$ функцией. Эти известные факты следуют, например, из [2, гл. VIII, раздел 83, теорема A].

Лемма 6. Пусть $x \in \Delta_{+}^{s} L_{p}(I)$, әде $s \in \mathbb{N}, 1 \leqslant p \leqslant \infty, a I:=(-1,1)$. Пусть

$$
\pi_{s}(t ; x):=\sum_{k=0}^{s-1} \frac{x^{(k)}(0)}{k !} t^{k}, \quad t \in I .
$$

Тогда для функиии

$$
\tilde{x}(t):=x(t)-\pi_{s}(t ; x), \quad t \in I,
$$

будет справедливо неравенство

$$
\|\tilde{x}\|_{L_{p}(I)} \leqslant \tilde{c}\|x\|_{L_{p}(I)}
$$

əде $\tilde{c}=\tilde{c}(s, p)$. 
3. Доказательство оценок снизу в теореме 1. Для того, чтобы оценить снизу поперечники $d_{n}\left(\Delta_{+}^{s} B_{p}\right)_{L_{p}}^{p s d}$ на интервале $I:=(-1,1)$, будем строить некоторые конечномерные множества, принадлежащие классам $\Delta_{+}^{s} B_{p}(I)$, а затем оценивать снизу псевдо-размерностные поперечники этих множеств.

Если $s \in \mathbb{N}$ и $1 \leqslant p \leqslant \infty$, то полагаем

$$
\chi_{s, p}(t ; \tau):=(s p+1)^{1 / p}(1-\tau)^{-s-1 / p}(t-\tau)_{+}^{s}, \quad \tau, t \in I
$$

где $(s \infty+1)^{1 / \infty}:=1$ и $(t-\tau)_{+}:=\max \{t-\tau, 0\}$. Ясно, что $\chi_{s, p}(\cdot ; \tau) \in \Delta_{+}^{s} B_{p}(I)$ при любом фиксированном $\tau \in I$. Очевидно, что функции $\chi_{s, p}(\cdot ; \tau)$ являются неотрицательными на $I$, и строго возрастают на промежутке $[\tau, 1)$. Кроме того,

$$
\left\|\chi_{s, p}(\cdot ; \tau)\right\|_{L_{p}(I)}=1, \quad \tau \in I, \quad 1 \leqslant p \leqslant \infty
$$

Рассмотрим вначале случай, когда $s \in \mathbb{N}$ и $p=\infty$. Зафиксировав число $\varepsilon, 0<\varepsilon<1 / 2$, и полагая $\check{\tau}_{\varepsilon, 0}:=0$, найдем точку $\check{\tau}_{\varepsilon, 1}:=\check{\tau}_{s, \varepsilon, 1}$, решив уравнение

$$
\chi_{s, \infty}\left(\check{\tau}_{\varepsilon, 1} ; \check{\tau}_{\varepsilon, 0}\right)=1-\varepsilon .
$$

Затем найдем точку $\hat{\tau}_{\varepsilon, 1}:=\hat{\tau}_{s, \varepsilon, 1}$, решая уравнение

$$
\chi_{s, \infty}\left(\hat{\tau}_{\varepsilon, 1} ; \check{\tau}_{\varepsilon, 1}\right)=\varepsilon .
$$

Точку $\check{\tau}_{\varepsilon, 2}:=\check{\tau}_{s, \varepsilon, 2}$ найдем из уравнения

$$
\chi_{s, \infty}\left(\check{\tau}_{\varepsilon, 2} ; \check{\tau}_{\varepsilon, 1}\right)=1-\varepsilon,
$$

а точку $\hat{\tau}_{\varepsilon, 2}:=\hat{\tau}_{s, \varepsilon, 2}-$ из уравнения

$$
\chi_{s, \infty}\left(\hat{\tau}_{\varepsilon, 2} ; \check{\tau}_{\varepsilon, 2}\right)=\varepsilon
$$

Продолжая этот процесс, найдем две последовательности точек $\left\{\check{\tau}_{\varepsilon, i}\right\}_{i=0}^{\infty}$ и $\left\{\hat{\tau}_{\varepsilon, i}\right\}_{i=1}^{\infty}$ таких, что $\check{\tau}_{\varepsilon, 0}<\check{\tau}_{\varepsilon, 1}<\hat{\tau}_{\varepsilon, 1}<\check{\tau}_{\varepsilon, 2}<\hat{\tau}_{\varepsilon, 2}<\cdots<\check{\tau}_{\varepsilon, i}<\hat{\tau}_{\varepsilon, i}<\cdots<1$.

При каждом $m \in \mathbb{N}$ полагаем

$$
\psi_{\varepsilon, i}(t):=\psi_{s, \varepsilon, m, i}(t):=\chi_{s, \infty}\left(t ; \check{\tau}_{\varepsilon, i-1}\right), \quad i=1, \ldots, m, \quad \psi_{\varepsilon, m+1}(t):=0, \quad t \in I .
$$

Очевидно, что $\psi_{\varepsilon, i} \in \Delta_{+}^{s} B_{\infty}(I), i=1, \ldots, m+1$. Кроме того, эти функции являются неотрицательными и неубывающими на $I$.

$\mathrm{C}$ помощью функций $\psi_{\varepsilon, i}$ определяем функции

$$
\xi_{\varepsilon, i}(t):=\xi_{s, \varepsilon, m, i}(t):=(2 m)^{-1}\left(\psi_{\varepsilon, i}(t)-\psi_{\varepsilon, i+1}(t)\right), \quad t \in I, \quad i=1, \ldots, m,
$$

и множество

$$
\Xi_{s, \varepsilon}^{m}:=\Xi_{s, \varepsilon}^{m}(I):=\left\{\xi_{\varepsilon, v} \mid \xi_{\varepsilon, v}:=\sum_{i=1}^{m} v_{i} \xi_{\varepsilon, i}, v:=\left(v_{1}, \ldots, v_{m}\right) \in\{-1,1\}^{m}\right\}
$$


состоящее из $2^{m}$ функций $\xi_{\varepsilon, v}, v \in\{-1,1\}^{m}$. Считая, что $m>1$, и полагая

$$
x_{\varepsilon, m}(t):=x_{s, \varepsilon, m}(t):=(2 m)^{-1} \psi_{\varepsilon, 1}(t)+m^{-1} \sum_{i=2}^{m} \psi_{\varepsilon, i}(t), \quad t \in I,
$$

рассмотрим на $I$ следующее множество:

$$
x_{\varepsilon, m}+\Xi_{s, \varepsilon}^{m}:=\left\{\zeta_{\varepsilon, v} \mid \zeta_{\varepsilon, v}:=x_{\varepsilon, m}+\xi_{\varepsilon, v}, v \in\{-1,1\}^{m}\right\},
$$

состоящее из $2^{m}$ функций $\zeta_{\varepsilon, v}, v \in\{-1,1\}^{m}$.

Убедимся, что все функции $\zeta_{\varepsilon, v}=x_{\varepsilon, m}+\xi_{\varepsilon, v}, v:=\left(v_{1}, \ldots, v_{m}\right) \in\{-1,1\}^{m}$, принадлежат классу $\Delta_{+}^{s} B_{\infty}(I)$. Действительно, учитывая определения функций $x_{\varepsilon, m}$ и $\xi_{\varepsilon, v}$, имеем

$$
x_{\varepsilon, m}(t)+\xi_{\varepsilon, v}(t)=(2 m)^{-1}\left(1+v_{1}\right) \psi_{\varepsilon, 1}(t)+(2 m)^{-1} \sum_{i=2}^{m}\left(2-v_{i-1}+v_{i}\right) \psi_{\varepsilon, i}(t) .
$$

Очевидно, что $1+v_{1} \geqslant 0$ и $2-v_{i-1}+v_{i} \geqslant 0, i=2, \ldots, m$. А так как $\psi_{\varepsilon, i} \in \Delta_{+}^{s} B_{\infty}(I)$, $i=1, \ldots, m$, то каждая функция $x_{\varepsilon, m}+\xi_{\varepsilon, v}$ является $s$-монотонной на $I$. Ясно также, что

$$
\begin{aligned}
\left\|x_{\varepsilon, m}+\xi_{\varepsilon, v}\right\|_{L_{\infty}(I)} & \leqslant(2 m)^{-1}\left(1+v_{1}\right)+(2 m)^{-1} \sum_{i=2}^{m}\left(2-v_{i-1}+v_{i}\right) \\
& =(2 m)^{-1}\left(2 m-1+v_{m}\right) \leqslant 1 .
\end{aligned}
$$

Поэтому все функции $x_{\varepsilon, m}+\xi_{\varepsilon, v}, v \in\{-1,1\}^{m}$, принадлежат классу $\Delta_{+}^{s} B_{\infty}(I)$.

Таким образом, доказано, что множество $x_{\varepsilon, m}+\Xi_{s, \varepsilon}^{m}$ содержится в $\Delta_{+}^{s} B_{\infty}(I)$. Но тогда

$$
d_{n}\left(\Delta_{+}^{s} B_{\infty}\right)_{L_{\infty}}^{p s d} \geqslant d_{n}\left(x_{\varepsilon, m}+\Xi_{s, \varepsilon}^{m}\right)_{L_{\infty}}^{p s d}, \quad n \geqslant 1 .
$$

Теперь воспользуемся тем обстоятельством, что сдвиг любого функционального класса на произвольную фиксированную функцию не меняет его псевдо-размерности. Для непустых подмножеств $W:=W(I)$ и $M:=M(I)$ из $L_{p}:=L_{p}(I), 1 \leqslant p \leqslant \infty$, будем обозначать через

$$
E(W, M)_{L_{p}}:=\sup _{x \in W} \inf _{y \in M}\|x-y\|_{L_{p}}
$$

величину наилучшего приближения в $L_{p}$ множества $W$ множеством $M$.

Пусть $M^{n}:=M^{n}(I)$ - произвольное многообразие функций из $L_{\infty}$ такое, что $\operatorname{dim}_{p s}\left(M^{n}\right) \leqslant n$. Очевидно, что

$$
E\left(x_{\varepsilon, m}+\Xi_{s, \varepsilon}^{m}, M^{n}\right)_{L_{\infty}}=E\left(\Xi_{s, \varepsilon}^{m},-x_{\varepsilon, m}+M^{n}\right)_{L_{\infty}} .
$$

Воспользовавшись утверждением 1) леммы 2, имеем

$$
\operatorname{dim}_{p s}\left(-x_{\varepsilon, m}+M^{n}\right)=\operatorname{dim}_{p s}\left(M^{n}\right) .
$$

Но тогда в силу произвольности выбора $M^{n}$ из (3.4) и (3.5) следует, что

$$
d_{n}\left(x_{\varepsilon, m}+\Xi_{s, \varepsilon}^{m}\right)_{L_{\infty}}^{p s d} \geqslant d_{n}\left(\Xi_{s, \varepsilon}^{m}\right)_{L_{\infty}}^{p s d} .
$$


Докажем, что если $m:=n+1$ и $0<\varepsilon<(n+2)^{-1}$, где $n \in \mathbb{N}$, то

$$
d_{n}\left(\Xi_{s, \varepsilon}^{n+1}\right)_{L_{\infty}}^{p s d} \geqslant(2 n+2)^{-1}(1-(n+2) \varepsilon) .
$$

Допустим от противного, что при некотором $n \in \mathbb{N}$ неравенство (3.7) не вьполняется. Тогда должно существовать множество $M^{n}=M^{n}(I)$ функций такое, что $\operatorname{dim}_{p s}\left(M^{n}\right) \leqslant n$ и

$$
E\left(\Xi_{s, \varepsilon}^{n+1}, M^{n}\right)_{L_{\infty}}<(2 n+2)^{-1}(1-(n+2) \varepsilon) .
$$

А следовательно, для каждой функции $\xi_{\varepsilon, v}$, где $v \in\{-1,1\}^{n+1}$, должна найтись функция $\mu\left(\cdot ; \xi_{\varepsilon, v}\right) \in M^{n}$ такая, что

$$
\left\|\xi_{\varepsilon, v}(\cdot)-\mu\left(\cdot ; \xi_{\varepsilon, v}\right)\right\|_{L_{\infty}(I)}<(2 n+2)^{-1}(1-(n+2) \varepsilon)
$$

Полагая

$$
I_{\varepsilon, i}:=I_{s, \varepsilon, i}:=\left(\check{\tau}_{\varepsilon, i}, \hat{\tau}_{\varepsilon, i}\right), \quad i=1, \ldots, n+1,
$$

отметим, что из определений функций $\psi_{\varepsilon, i}$ и $\xi_{\varepsilon, i}$ следуют неравенства

$$
\begin{gathered}
\xi_{\varepsilon, i}(t) \geqslant(2 n+2)^{-1}(1-2 \varepsilon), \quad t \in I_{\varepsilon, i}, \quad i=1, \ldots, n+1 \\
0 \leqslant \xi_{\varepsilon, i}(t) \leqslant(2 n+2)^{-1} \varepsilon, \quad t \in I_{\varepsilon, j}, \quad i, j=1, \ldots, n+1, \quad j \neq i
\end{gathered}
$$

Поэтому при любом $v:=\left(v_{1}, \ldots, v_{n+1}\right) \in\{-1,1\}^{n+1}$ будут верны неравенства

$$
\xi_{\varepsilon, v}(t) \operatorname{sgn} v_{i} \geqslant(2 n+2)^{-1}(1-(n+2) \varepsilon)>0, \quad t \in I_{\varepsilon, i}, \quad i=1, \ldots, n+1 .
$$

Но из этих неравенств и (3.8) будет следовать, что

$$
\operatorname{sgn} \mu\left(t ; \xi_{\varepsilon, v}\right)=\operatorname{sgn} v_{i}, \quad \text { п. в. } t \in I_{\varepsilon, i}, \quad i=1, \ldots, n+1 .
$$

А из этих равенств и определения псевдо-размерности сразу же следует неравенство $\operatorname{dim}_{p s}\left(M^{n}\right) \geqslant n+1$, хотя предполагалось, что $\operatorname{dim}_{p s}\left(M^{n}\right) \leqslant n$. Полученное противоречие доказьвает справедливость неравенства (3.7).

Число $\varepsilon$ может быть выбрано сколь угодно малым. Поэтому из (3.3), (3.6) и (3.7) следует, что

$$
d_{n}\left(\Delta_{+}^{s} B_{\infty}\right)_{L_{\infty}}^{p s d} \geqslant 4^{-1} n^{-1}, \quad n \geqslant 1, \quad s \in \mathbb{N} .
$$

Таким образом, в случае $p=\infty$ необходимая оценка снизу в соотношениях (1.1) и (1.2) для псевдо-размерностных поперечников доказана.

Рассмотрим теперь случай, когда $s \in \mathbb{N}$ и $1 \leqslant p<\infty$. Зафиксировав число $\varepsilon$, $0<\varepsilon<1$, и полагая $\tau_{\varepsilon, 0}^{*}:=\tau_{s, p, \varepsilon, 0}^{*}:=0$, определим точки $\tau_{\varepsilon, i}^{*}:=\tau_{s, p, \varepsilon, i}^{*}, i=1,2, \ldots$, решая одно за другим уравнения

$$
\left\|\chi_{s, p}\left(\cdot ; \tau_{\varepsilon, i-1}^{*}\right)\right\|_{L_{p}\left[\tau_{\varepsilon, i}^{*}, 1\right)}=\varepsilon, \quad i=1,2, \ldots,
$$

где $\chi_{s, p}(\cdot ; \tau)$ - функция из (3.1). Ясно, что полученная последовательность $\left\{\tau_{\varepsilon, i}^{*}\right\}_{i=0}^{\infty}$ строго возрастает, т.е. $0=\tau_{\varepsilon, 0}^{*}<\tau_{\varepsilon, 1}^{*}<\cdots<\tau_{\varepsilon, i}^{*}<\cdots<1$. Нетрудно также проверить, что

$$
\tau_{\varepsilon, i}^{*}=\tau_{\varepsilon, i-1}^{*}+\left(1-\varepsilon^{p}\right)^{1 /(s p+1)}\left(1-\tau_{\varepsilon, i-1}^{*}\right), \quad i \in \mathbb{N} .
$$


Полагая

$$
I_{\varepsilon, i}^{*}:=I_{s, p, \varepsilon, i}^{*}:=\left[\tau_{\varepsilon, i-1}^{*}, 1\right), \quad i=1,2, \ldots,
$$

определим при каждом $m>1$ следующие промежутки

$$
\begin{aligned}
I_{\varepsilon, i}^{\star} & :=I_{s, p, \varepsilon, m, i}^{\star}:=\left[\tau_{\varepsilon, i-1}^{*}, \tau_{\varepsilon, i}^{*}\right), \quad i=1, \ldots, m-1, \\
I_{\varepsilon, m}^{\star} & :=I_{s, p, \varepsilon, m, m}^{\star}:=\left[\tau_{\varepsilon, m-1}^{*}, 1\right) .
\end{aligned}
$$

Очевидно, что $I_{\varepsilon, i}^{\star} \subseteq I_{\varepsilon, i}^{*}, i=1, \ldots, m$. Отметим, что в силу (3.11) имеют место равенства

$$
\left|I_{\varepsilon, i}^{\star}\right|=\left(1-\varepsilon^{p}\right)^{1 /(s p+1)}\left|I_{\varepsilon, i}^{*}\right|, \quad i=1, \ldots, m-1, \quad\left|I_{\varepsilon, m}^{\star}\right|=\left|I_{\varepsilon, m}^{*}\right| .
$$

Определим теперь при каждом $m>1$ следующие функции:

$$
y_{\varepsilon, m}(t):=y_{s, p, \varepsilon, m}(t):=2^{-2} m^{-1 / p} \sum_{i=1}^{m} \chi_{s, p}\left(t ; \tau_{\varepsilon, i-1}^{*}\right), \quad t \in I, \quad m \in \mathbb{N},
$$

и оценим сверху их нормы в $L_{p}(I)$. Очевидно, что

$$
y_{\varepsilon, m}(t)=2^{-2} m^{-1 / p} \sum_{j=1}^{i} \chi_{s, p}\left(t ; \tau_{\varepsilon, j-1}^{*}\right), \quad t \in I_{\varepsilon, i}^{\star}, \quad i=1, \ldots, m .
$$

Кроме того, если $1<i \leqslant m$, то будут справедливы соотношения

$$
\begin{aligned}
& \int_{I_{\varepsilon, i}^{\star}}\left(\sum_{j=1}^{i} \chi_{s, p}\left(t ; \tau_{\varepsilon, j-1}^{*}\right)\right)^{p} d t \\
& \quad \leqslant 2^{p-1} \int_{I_{\varepsilon, i}^{\star}}\left(\sum_{j=1}^{i-1} \chi_{s, p}\left(t ; \tau_{\varepsilon, j-1}^{*}\right)\right)^{p} d t+2^{p-1} \int_{I_{\varepsilon, i}^{\star}}\left(\chi_{s, p}\left(t ; \tau_{\varepsilon, i-1}^{*}\right)\right)^{p} d t \\
& \leqslant 2^{p-1}(i-1)^{p-1} \sum_{j=1}^{i-1} \int_{I_{\varepsilon, j+1}^{*}}\left(\chi_{s, p}\left(t ; \tau_{\varepsilon, j-1}^{*}\right)\right)^{p} d t+2^{p-1} \int_{I_{\varepsilon, i}^{*}}\left(\chi_{s, p}\left(t ; \tau_{\varepsilon, i-1}^{*}\right)\right)^{p} d t \\
& =2^{p-1}\left((i-1)^{p} \varepsilon^{p}+1\right) .
\end{aligned}
$$

В этих оценках кроме известного неравенства $\left(\sum_{i=1}^{n}\left|a_{i}\right|\right)^{p} \leqslant n^{p-1} \sum_{i=1}^{n}\left|a_{i}\right|^{p}$ использовались равенства (3.2) и (3.10). Отметим также, что

$$
\int_{I_{\varepsilon, 1}^{\star}}\left(\chi_{s, p}\left(t ; \tau_{\varepsilon, 0}^{*}\right)\right)^{p} d t \leqslant \int_{I_{\varepsilon, 1}^{*}}\left(\chi_{s, p}\left(t ; \tau_{\varepsilon, 0}^{*}\right)\right)^{p} d t=1
$$

Учитьвая полученные оценки и равенства (3.14), приходим к неравенству

$$
\left\|y_{\varepsilon, m}\right\|_{L_{p}(I)} \leqslant 2^{-1-1 / p}\left(1+(m \varepsilon)^{p}\right)^{1 / p}
$$

Построим теперь на интервале $I$ некоторые множества $\Phi_{s, \varepsilon}^{m}:=\Phi_{s, p, \varepsilon}^{m}(I)$ функций, связанные с разбиениями промежутка $[0,1)$ на промежутки $I_{\varepsilon, i}^{\star}$. Полагая

$$
\phi_{0}(t):= \begin{cases}1 & \text { для } t \in(-1,1), \\ 0 & \text { для } t \in \mathbb{R} \backslash(-1,1),\end{cases}
$$


рассмотрим функции

$$
\phi_{s}(t):=\int_{-\infty}^{t}\left(\phi_{s-1}(2 \tau+1)-\phi_{s-1}(2 \tau-1)\right) d \tau, \quad t \in \mathbb{R}, \quad s \in \mathbb{N} .
$$

Ясно, что $\operatorname{supp} \phi_{s}=[-1,1]$. Нетрудно также проверить, что при $s \in \mathbb{N}$ имеют место равенства

$$
\left\|\phi_{s}\right\|_{L_{\infty}(I)}=2^{-s+1}, \quad\left\|\phi_{s}\right\|_{L_{1}(I)}=2^{-s+1}, \quad\left\|\phi_{s}^{(s)}\right\|_{L_{\infty}(I)}=2^{(s-1) s / 2} .
$$

Обозначая через $\bar{\tau}_{\varepsilon, i}^{\star}$ середины промежутков $I_{\varepsilon, i}^{\star}$, определим на интервале $I$ при $i=1$, .., $m$ следуюшие функции:

$$
\varphi_{\varepsilon, i}(t):=\varphi_{s, p, \varepsilon, m, i}(t):=\frac{\left|I_{\varepsilon, i}^{\star}\right|^{s}}{2^{s(s+1) / 2+2} m^{1 / p}\left|I_{\varepsilon, i}^{*}\right|^{s+1 / p}} \phi_{s}\left(\frac{2\left(t-\bar{\tau}_{\varepsilon, i}^{\star}\right)}{\left|I_{\varepsilon, i}^{\star}\right|}\right) .
$$

Очевидно, что каждая из функций $\varphi_{\varepsilon, i}$ неотрицательна на соответствующем промежутке $I_{\varepsilon, i}^{\star}$, а ее носитель совпадает с замыканием этого промежутка. Воспользовавшись равенствами (3.17), нетрудно проверить, что при $s \in \mathbb{N}$ справедливы равенства

$$
\left\|\varphi_{\varepsilon, i}\right\|_{L_{\infty}\left(I_{\varepsilon, i}^{\star}\right)}=2^{-s(s+3) / 2-1} m^{-1 / p}\left|I_{\varepsilon, i}^{*}\right|^{-s-1 / p}\left|I_{\varepsilon, i}^{\star}\right|^{s}, \quad i=1, \ldots, m,
$$

и

$$
\left\|\varphi_{\varepsilon, i}^{(s)}\right\|_{L_{\infty}\left(I_{\varepsilon, i}^{\star}\right)}=2^{-2} m^{-1 / p}\left|I_{\varepsilon, i}^{*}\right|^{-s-1 / p}, \quad i=1, \ldots, m .
$$

Кроме того, из (3.19) следуют неравенства

$$
\left\|\varphi_{\varepsilon, i}\right\|_{L_{p}\left(I_{\varepsilon, i}^{\star}\right)} \leqslant 2^{-s(s+3) / 2-1} m^{-1 / p}\left|I_{\varepsilon, i}^{*}\right|^{-s-1 / p}\left|I_{\varepsilon, i}^{\star}\right|^{s+1 / p}, \quad i=1, \ldots, m .
$$

$\mathrm{C}$ помощью функций $\varphi_{\varepsilon, i}$ определяем на интервале $I$ множества

$$
\Phi_{s, \varepsilon}^{m}:=\Phi_{s, \varepsilon}^{m}(I):=\left\{\varphi_{\varepsilon, v} \mid \varphi_{\varepsilon, v}:=\sum_{i=1}^{m} v_{i} \varphi_{\varepsilon, i}, v:=\left(v_{1}, \ldots, v_{m}\right) \in\{-1,1\}^{m}\right\}
$$

каждое из которых состоит из $2^{m}$ функций.

Полагая

$$
y_{\varepsilon, m}+\Phi_{s, \varepsilon}^{m}:=\left\{\zeta_{\varepsilon, v} \mid \zeta_{\varepsilon, v}:=y_{\varepsilon, m}+\varphi_{\varepsilon, v}, v:=\left(v_{1}, \ldots, v_{m}\right) \in\{-1,1\}^{m}\right\},
$$

где $y_{\varepsilon, m}$ определены в $(3.13)$, убедимся, что при условии $0<\varepsilon \leqslant m^{-1}$ эти множества функций принадлежат классу $\Delta_{+}^{s} B_{p}(I)$.

Пусть $y_{\varepsilon, m}+\varphi_{\varepsilon, v}-$ произвольная функция из множества $y_{\varepsilon, m}+\Phi_{s, \varepsilon}^{m}$. Тогда, учитывая $(3.15)$ и $(3.21)$, получаем

$$
\begin{aligned}
\left\|y_{\varepsilon, m}+\varphi_{\varepsilon, v}\right\|_{L_{p}(I)} & \leqslant\left\|y_{\varepsilon, m}\right\|_{L_{p}(I)}+\left(\sum_{i=1}^{m}\left\|\varphi_{\varepsilon, i}\right\|_{L_{p}\left(I_{\varepsilon, i}^{\star}\right)}^{p}\right)^{1 / p} \\
& \leqslant 2^{-1}+2^{-s(s+3) / 2-1} m^{-1 / p}\left(\sum_{i=1}^{m}\left|I_{\varepsilon, i}^{*}\right|^{-s p-1}\left|I_{\varepsilon, i}^{\star}\right|^{s p+1}\right)^{1 / p} \\
& \leqslant 2^{-1}+2^{-s(s+3) / 2-1} \leqslant 1 .
\end{aligned}
$$


Следовательно, функция $y_{\varepsilon, m}+\varphi_{\varepsilon, v}$ принадлежит единичному шару $B_{p}(I)$. Отметим теперь, что из (3.2) и (3.14) при всех $i=1, \ldots, m$ следуют равенства

$$
y_{\varepsilon, m}^{(s)}(t)=2^{-2} s !(s p+1)^{1 / p} m^{-1 / p} \sum_{j=1}^{i}\left|I_{\varepsilon, j}^{*}\right|^{-s-1 / p}, \quad t \in\left(\tau_{\varepsilon, i-1}^{*}, \tau_{\varepsilon, i}^{*}\right) .
$$

Дифференцируя функцию $y_{\varepsilon, m}+\varphi_{\varepsilon, v}$ и учитьвая соотношения $(3.20)$ и $(3.22)$, видим, что для почти всех $t \in I_{\varepsilon, i}^{\star}$, где $i=1, \ldots, m$, имеют место соотношения

$$
\begin{aligned}
y_{\varepsilon, m}^{(s)}(t)+\varphi_{\varepsilon, v}^{(s)}(t) & \geqslant 2^{-2} s !(s p+1)^{1 / p} m^{-1 / p} \sum_{j=1}^{i}\left|I_{\varepsilon, j}^{*}\right|^{-s-1 / p}-\left\|\varphi_{\varepsilon, i}^{(s)}\right\|_{L_{\infty}\left(I_{\varepsilon, i}^{\star}\right)} \\
& \geqslant 2^{-2} m^{-1 / p}\left|I_{\varepsilon, i}^{*}\right|^{-s-1 / p}-2^{-2} m^{-1 / p}\left|I_{\varepsilon, i}^{*}\right|^{-s-1 / p}=0 .
\end{aligned}
$$

Следовательно, функция $y_{\varepsilon, m}+\varphi_{\varepsilon, v}$ имеет почти всюду на $I$ неотрицательную (кусочно-постоянную) производную порядка $s$. Поэтому эта функция является $s$-монотонной на $I$. Таким образом, установлено, что множество $y_{\varepsilon, m}+\Phi_{s, \varepsilon}^{m}$ принадлежит классу $\Delta_{+}^{s} B_{p}(I)$.

Но тогда очевидно, что при условии $0<\varepsilon \leqslant m^{-1}$ имеет место оценка

$$
d_{n}\left(\Delta_{+}^{s} B_{p}\right)_{L_{p}}^{p s d} \geqslant d_{n}\left(y_{\varepsilon, m}+\Phi_{s, \varepsilon}^{m}\right)_{L_{p}}^{p s d} .
$$

Воспользовавшись затем утверждением 1) леммы 2, приходим к неравенству

$$
d_{n}\left(y_{\varepsilon, m}+\Phi_{s, \varepsilon}^{m}\right)_{L_{p}}^{p s d} \geqslant d_{n}\left(\Phi_{s, \varepsilon}^{m}\right)_{L_{p}}^{p s d} .
$$

Задачу об оценке снизу в $L_{p}$ поперечников множеств $\Phi_{s, \varepsilon}^{m}$ сведем к задаче об оценке снизу в $L_{1}$ поперечников других конечномерных множеств $\Phi_{s}^{m}$. Для этого разобьем промежуток $[0,1)$ точками $\tau_{i}:=\tau_{m, i}:=i / m, i=0,1, \ldots, m$, на $m$ промежутков $I_{i}:=$ $I_{m, i}:=\left[\tau_{i-1}, \tau_{i}\right), i=1, \ldots, m$, равной длины, и обозначим через $\bar{\tau}_{i}, i=1, \ldots, m$, середины этих промежутков. Определим затем функции

$$
\varphi_{i}(t):=\varphi_{s, m, i}(t):=2^{-s(s+1) / 2-2} \phi_{s}\left(2 m\left(t-\bar{\tau}_{i}\right)\right), \quad t \in I, \quad i=1, \ldots, m,
$$

где $\phi_{s}-$ функции из (3.16). Очевидно, что носители функций $\varphi_{i}(t)$ совпадают с замыканиями соответствующих промежутков $I_{i}$.

Полагая

$$
\Phi_{s}^{m}:=\Phi_{s}^{m}(I):=\left\{\varphi_{v} \mid \varphi_{v}:=\sum_{i=1}^{m} v_{i} \varphi_{i}, v:=\left(v_{1}, \ldots, v_{m}\right) \in\{-1,1\}^{m}\right\},
$$

докажем, что

$$
d_{n}\left(\Phi_{s, \varepsilon}^{m}\right)_{L_{p}(I)}^{p s d} \geqslant\left(1-\varepsilon^{p}\right)^{1 / p} d_{n}\left(\Phi_{s}^{m}\right)_{L_{1}(0,1)}^{p s d} .
$$

Пусть $M^{n}:=M^{n}(I)$ - произвольное множество функций $\mu: I \rightarrow \mathbb{R}$ из $L_{p}(I)$ такое, что $\operatorname{dim}_{p s}\left(M^{n}\right) \leqslant n$. Очевидно, что для любых функций $\varphi_{\varepsilon, v} \in \Phi_{s, \varepsilon}^{m}$ и $\mu \in M^{n}$ имеют место соотношения

$$
\left\|\varphi_{\varepsilon, v}-\mu\right\|_{L_{p}(I)} \geqslant\left\|\varphi_{\varepsilon, v}-\mu\right\|_{L_{p}[0,1)}=\left(\sum_{i=1}^{m} \int_{I_{\varepsilon, i}^{\star}}\left|v_{i} \varphi_{\varepsilon, i}(t)-\mu(t)\right|^{p} d t\right)^{1 / p}
$$


где $v:=\left(v_{1}, \ldots, v_{m}\right) \in\{-1,1\}^{m}$, а функции $\varphi_{\varepsilon, i}$ определены в (3.18). Произведем замены переменных

$$
\theta=\bar{\tau}_{i}+m^{-1}\left|I_{\varepsilon, i}^{\star}\right|^{-1}\left(t-\bar{\tau}_{\varepsilon, i}^{\star}\right), \quad t \in I_{\varepsilon, i}^{\star}, \quad i=1, \ldots, m,
$$

где $\bar{\tau}_{i}$ - середины промежутков $I_{i}$, а $\bar{\tau}_{\varepsilon, i}^{\star}$ - середины промежутков $I_{\varepsilon, i}^{\star}$. Ясно, что при этом каждьй промежуток $I_{\varepsilon, i}^{\star}$ взаимно-однозначньм образом отображается на промежуток $I_{i}$. Учитьвая определения (3.18) и (3.25) соответственно функций $\varphi_{\varepsilon, i}$ и $\varphi_{i}$, a также равенства (3.12), получаем

$$
\begin{aligned}
& \int_{I_{\varepsilon, i}^{\star}}\left|v_{i} \varphi_{\varepsilon, i}(t)-\mu(t)\right|^{p} d t \\
& \quad=\left(\frac{\left|I_{\varepsilon, i}^{\star}\right|}{\left|I_{\varepsilon, i}^{*}\right|}\right)^{s p+1} \int_{I_{i}}\left|v_{i} \varphi_{i}(\theta)-\frac{m^{1 / p}\left|I_{\varepsilon, i}^{*}\right|^{s+1 / p}}{\left|I_{\varepsilon, i}^{\star}\right|^{s}} \mu\left(\bar{\tau}_{\varepsilon, i}^{\star}+m\left|I_{\varepsilon, i}^{\star}\right|\left(\theta-\bar{\tau}_{i}\right)\right)\right|^{p} d \theta \\
& \quad \geqslant\left(1-\varepsilon^{p}\right) \int_{I_{i}}\left|v_{i} \varphi_{i}(\theta)-\frac{m^{1 / p}\left|I_{\varepsilon, i}^{*}\right|^{s+1 / p}}{\left|I_{\varepsilon, i}^{\star}\right|^{s}} \mu\left(\bar{\tau}_{\varepsilon, i}^{\star}+m\left|I_{\varepsilon, i}^{\star}\right|\left(\theta-\bar{\tau}_{i}\right)\right)\right|^{p} d \theta .
\end{aligned}
$$

Рассмотрим функции $\mu_{\varepsilon}:[0,1) \rightarrow \mathbb{R}$, определяемые на каждом из промежутков $I_{i}, i=1$, $\ldots, m$, согласно равенствам

$$
\mu_{\varepsilon}(\theta):=\mu_{s, p, \varepsilon, m}(\theta):=\frac{m^{1 / p}\left|I_{\varepsilon, i}^{*}\right|^{s+1 / p}}{\left|I_{\varepsilon, i}^{\star}\right|^{s}} \mu\left(\bar{\tau}_{\varepsilon, i}^{\star}+m\left|I_{\varepsilon, i}^{\star}\right|\left(\theta-\bar{\tau}_{i}\right)\right), \quad \theta \in I_{i} .
$$

Тогда из полученных оценок для интегралов и соотношений (3.27) следует, что

$$
\left\|\varphi_{\varepsilon, v}-\mu\right\|_{L_{p}(I)} \geqslant\left(1-\varepsilon^{p}\right)^{1 / p}\left\|\varphi_{v}-\mu_{\varepsilon}\right\|_{L_{1}(0,1)} .
$$

Обозначая через $M_{\varepsilon}^{n}:=M_{s, p, \varepsilon, m}^{n}(0,1)$ множество всех функций $\mu_{\varepsilon}:(0,1) \rightarrow \mathbb{R}$, полученных из функций $\mu \in M^{n}(I)$ путем преобразований (3.28), приходим к неравенству

$$
E\left(\Phi_{s, \varepsilon}^{m}, M^{n}\right)_{L_{p}(I)} \geqslant\left(1-\varepsilon^{p}\right)^{1 / p} E\left(\Phi_{s}^{m}, M_{\varepsilon}^{n}\right)_{L_{1}(0,1)}
$$

Отметим, что в силу свойств псевдо-размерности 2)-4), указанных в лемме 2, будут справедливы неравенства $\operatorname{dim}_{p s}\left(M_{\varepsilon}^{n}\right) \leqslant \operatorname{dim}_{p s}\left(M^{n}\right) \leqslant n$. Поэтому из (3.29) в силу произвольности выбора множества $M^{n}$ следует оценка (3.26).

Докажем теперь, что при $m:=160 n$ будет справедлива оценка

$$
d_{n}\left(\Phi_{s}^{m}\right)_{L_{1}(0,1)}^{p s d} \geqslant 2^{-s(s+3) / 2-6}, \quad n \geqslant 1 .
$$

Пусть $V^{(m)}-$ множество векторов $v \in\{-1,1\}^{m}$ такое, что $\operatorname{card}\left(V^{(m)}\right) \geqslant 2^{m / 16}$, и при этом

$$
\|\hat{v}-\check{v}\|_{l_{1}^{m}} \geqslant \frac{m}{2}, \quad \hat{v}, \check{v} \in V^{(m)}, \quad \hat{v} \neq \check{v} .
$$

Существование множества $V^{(m)}$ гарантировано леммой 3. Затем полагаем

$$
\Phi_{s}^{(m)}:=\Phi_{s}^{(m)}(I):=\left\{\varphi_{v} \mid \varphi_{v}=\sum_{i=1}^{m} v_{i} \varphi_{i}, v:=\left(v_{1}, \ldots, v_{m}\right) \in V^{(m)}\right\} .
$$


А так как $\Phi_{s}^{(m)} \subset \Phi_{s}^{m}$, то очевидно, что

$$
d_{n}\left(\Phi_{s}^{m}\right)_{L_{1}(0,1)}^{p s d} \geqslant d_{n}\left(\Phi_{s}^{(m)}\right)_{L_{1}(0,1)}^{p s d} .
$$

Из (3.17) и (3.25) следует, что при всех $i=1, \ldots, m$ имеют место равенства

$$
\left\|\varphi_{i}\right\|_{L_{\infty}\left(I_{i}\right)}=2^{-s(s+3) / 2-1}, \quad\left\|\varphi_{i}\right\|_{L_{1}\left(I_{i}\right)}=2^{-s(s+3) / 2-2} m^{-1} .
$$

Но тогда из (3.33) следует, что

$$
\left\|\varphi_{v}\right\|_{L_{\infty}(0,1)}=2^{-s(s+3) / 2-1}, \quad \varphi_{v} \in \Phi_{s}^{(m)} .
$$

Кроме того, для любых двух функций $\varphi_{\hat{v}}, \varphi_{\check{v}} \in \Phi_{s}^{(m)}$ таких, что $\hat{v} \neq \check{v}$ в силу (3.31) и (3.33) будут справедливы соотношения

$$
\left\|\varphi_{\hat{v}}-\varphi_{\check{v}}\right\|_{L_{1}(0,1)}=2^{-s(s+3) / 2-2} m^{-1}\|\hat{v}-\check{v}\|_{l_{1}^{m}} \geqslant 2^{-s(s+3) / 2-3} .
$$

Учитьвая (3.34) и (3.35), применим к множеству $\Phi_{s}^{(m)}$ лемму 5 , полагая в ней $a:=$ $2^{-s(s+3) / 2-1}, \alpha:=2^{-s(s+3) / 2-3}$ и $m:=160 n$. После чего получаем

$$
d_{n}\left(\Phi_{s}^{(m)}\right)_{L_{1}(0,1)}^{p s d} \geqslant 2^{-s(s+3) / 2-6} .
$$

А из из этой оценки и (3.32) сразу же следует оценка (3.30).

Ясно, что при условии $0<\varepsilon \leqslant(160 n)^{-1}$ из $(3.23),(3.24),(3.26)$ и (3.30) следует оценка

$$
d_{n}\left(\Delta_{+}^{s} B_{p}\right)_{L_{p}}^{p s d} \geqslant\left(1-\varepsilon^{p}\right)^{1 / p} 2^{-s(s+3) / 2-6} .
$$

А так как в этом неравенстве $\varepsilon$ может быть сколь угодно мальм, то

$$
d_{n}\left(\Delta_{+}^{s} B_{p}\right)_{L_{p}}^{p s d} \geqslant 2^{-s(s+3) / 2-6}, \quad n \geqslant 1, \quad 1 \leqslant p<\infty, \quad s \in \mathbb{N} .
$$

Таким образом, в случае $1 \leqslant p<\infty$ доказана необходимая оценка снизу в (1.4) для псевдо-размерностных поперечников.

Остается доказать оценки снизу для наилучших приближений сплайнами и рациональными функциями. А это сделать легко.

В п. 1 уже отмечалось, что

$$
\operatorname{dim}_{p s}\left(\Sigma_{r, n}\right) \asymp \operatorname{dim}_{p s}\left(R_{n}\right) \asymp n, \quad n \geqslant 1 .
$$

Поэтому

$$
E\left(\Delta_{+}^{s} B_{p}, \Sigma_{r, n}\right)_{L_{p}} \geqslant c d_{n}\left(\Delta_{+}^{s} B_{p}\right)_{L_{p}}^{p s d}, \quad n \geqslant 1, \quad 1 \leqslant p \leqslant \infty, \quad r, s \in \mathbb{N},
$$

и

$$
E\left(\Delta_{+}^{s} B_{p}, R_{n}\right)_{L_{p}} \geqslant c d_{n}\left(\Delta_{+}^{s} B_{p}\right)_{L_{p}}^{p s d}, \quad n \geqslant 1, \quad 1 \leqslant p \leqslant \infty, \quad s \in \mathbb{N}
$$

где $c>0$ не зависит от $n$.

Но тогда в силу (3.37) из (3.9) и (3.36) следуют, соответственно, оценки

$$
E\left(\Delta_{+}^{s} B_{\infty}, \Sigma_{r, n}\right) L_{\infty} \geqslant c n^{-1}, \quad n \geqslant 1, \quad r, s \in \mathbb{N},
$$


и

$$
E\left(\Delta_{+}^{s} B_{p}, \Sigma_{r, n}\right)_{L_{p}} \geqslant c, \quad n \geqslant 1, \quad 1 \leqslant p<\infty, \quad r, s \in \mathbb{N}
$$

где $c>0$ не зависит от $n$. Итак, для сплайнов необходимые оценки снизу в $(1.1),(1.2)$ и (1.4) доказаны.

Кроме того, воспользовавшись оценками (3.38), (3.9) и (3.36), имеем

$$
E\left(\Delta_{+}^{s} B_{\infty}, R_{n}\right)_{L_{\infty}} \geqslant c n^{-1}, \quad n \geqslant 1, s \in \mathbb{N}, \quad s>1,
$$

И

$$
E\left(\Delta_{+}^{s} B_{p}, R_{n}\right)_{L_{p}} \geqslant c, \quad n \geqslant 1, \quad 1 \leqslant p<\infty, \quad s \in \mathbb{N},
$$

где $c>0$ также не зависит от $n$. Таким образом, необходимые оценки снизу в соотношениях (1.1) и (1.4) получены и для рациональных функций.

Оценка же

$$
E\left(\Delta_{+}^{1} B_{\infty}, R_{n}\right)_{L_{\infty}} \geqslant c, \quad n \geqslant 1,
$$

в (1.3) является тривиальной, так как функция $z(t):=\operatorname{sgn} t, t \in I$, принадлежит классу $\Delta_{+}^{1} B_{\infty}$, но ее наилучшие приближения в $L_{\infty}$ множествами $R_{n}$ равны $1 / 2$ при всех $n \geqslant 1$.

Доказательство оценок снизу в теореме 1 закончено.

4. Доказательство оценок сверху в теореме 1 . Пусть $I:=(-1,1), s \in \mathbb{N}$ и $p=\infty$. Будем вначале рассматривать функции $\tilde{x} \in \Delta_{+}^{s} L_{\infty}(I)$, удовлетворяющие условиям

$$
\tilde{x}^{(k)}(0)=0, \quad k=0, \ldots, s-1 .
$$

Отметим, что, в силу монотонности производной $\tilde{x}^{(s-1)}$, из $(4.1)$ следуют неравенства $\tilde{x}^{(k)}(t) \geqslant 0$ для $t \in I_{+}:=[0,1)$, где $k=0, \ldots, s-1$. Если же $t \in I_{-}:=(-1,0]$, то $(-1)^{s-k} \tilde{x}^{(k)}(t) \geqslant 0$, где $k=0, \ldots, s-1$. При $s=1$ это очевидно, а при $s>1$ легко проверяется с помощью формулы Тейлора.

Функция $\tilde{x}$ является ограниченной и монотонной на каждом из промежутков $I_{+}$и $I_{-}$. Поэтому существуют сплайны $\sigma_{1, n}(\cdot ; \tilde{x} ; I) \in \Sigma_{1, n}(I)$ такие, что

$$
\left\|\tilde{x}(\cdot)-\sigma_{1, n}(\cdot ; \tilde{x} ; I)\right\|_{L_{\infty}(I)} \leqslant\|\tilde{x}\|_{L_{\infty}(I)} n^{-1}, \quad n \geqslant 1, \quad s \in \mathbb{N} .
$$

Если $s>1$, то полагая $\tilde{x}_{+}(t):=\max \{0, \tilde{x}(t)\}$ и $\tilde{x}_{-}(t):=\max \left\{0,(-1)^{s} \tilde{x}(t)\right\}$ для $t \in I$, отметим, что обе функции $\tilde{x}_{+}$и $\tilde{x}_{-}$являются вьпукльми на $I$. Поэтому, воспользовавшись результатом Попова и Петрушева [5], можем утверждать, что при каждом $n \in \mathbb{N}$ существуют рациональные функции $\rho_{n}\left(\cdot ; \tilde{x}_{+}\right), \rho_{n}\left(\cdot ; \tilde{x}_{-}\right) \in R_{n}(I)$ такие, что

$$
\left\|\tilde{x}_{+}(\cdot)-\rho_{n}\left(\cdot ; \tilde{x}_{+} ; I\right)\right\|_{L_{\infty}(I)} \leqslant c\left\|\tilde{x}_{+}\right\|_{L_{\infty}(I)} n^{-1}
$$

и

$$
\left\|\tilde{x}_{-}(\cdot)-\rho_{n}\left(\cdot ; \tilde{x}_{-} ; I\right)\right\|_{L_{\infty}(I)} \leqslant c\left\|\tilde{x}_{-}\right\|_{L_{\infty}(I)} n^{-1}
$$

где $c>0$ не зависит от $n$. Но тогда существуют $\rho_{2 n}(\cdot ; \tilde{x}) \in R_{2 n}(I)$ такие, что

$$
\left\|\tilde{x}(\cdot)-\rho_{2 n}(\cdot ; \tilde{x} ; I)\right\|_{L_{\infty}(I)} \leqslant c\|\tilde{x}\|_{L_{\infty}(I)} n^{-1}, \quad n \geqslant 1, \quad s \in \mathbb{N}, \quad s>1,
$$

где $c>0$ не зависит от $n$. 
Оценки (4.2) и (4.3) установлены для функций $\tilde{x} \in \Delta_{+}^{s} L_{\infty}(I)$, удовлетворяющих условиям (4.1). Чтобы избавиться от ограничений (4.1), поступим следующим образом.

Для каждой функции $x \in \Delta_{+}^{s} L_{\infty}(I)$ обозначим через

$$
\pi_{s}(t ; x):=\sum_{k=0}^{s-1} \frac{x^{(k)}(0)}{k !} t^{k}, \quad t \in I
$$

многочлен Тейлора порядка $\leqslant s$, построенньй относительно точки $t=0$. Затем полагаем

$$
\tilde{x}(t):=x(t)-\pi_{s}(t ; x), \quad t \in I .
$$

Ясно, что эта функция удовлетворяет условиям (4.1). Кроме того, из леммы 6 следует, что $\|\tilde{x}\|_{L_{\infty}(I)} \leqslant \tilde{c}\|x\|_{L_{\infty}(I)}$, где $\tilde{c}=\tilde{c}(s)$.

Если $r \geqslant s$, то для функций $x \in \Delta_{+}^{s} B_{\infty}(I)$ приближающие сплайны определяем, полагая

$$
\sigma_{r, n}(t ; x ; I):=\sigma_{1, n}(t ; \tilde{x} ; I)+\pi_{s}(t ; x), \quad t \in I .
$$

Тогда $\sigma_{r, n}(\cdot ; x ; I) \in \Sigma_{r, n}(I)$, а из (4.2) будет следовать, что

$$
\left\|x(\cdot)-\sigma_{r, n}(\cdot ; x ; I)\right\|_{L_{\infty}(I)} \leqslant c n^{-1}, \quad n \geqslant 1, \quad r, s \in \mathbb{N}, \quad r \geqslant s,
$$

где $c=c(s)$.

Если же $r<s$, то сплайны вида (4.4), вообще говоря, не принадлежат $\Sigma_{r, n}(I)$. Однако их легко подправить, заменив многочлены $\pi_{s}(x):=\pi_{s}(\cdot ; x)$ на сплайны $\sigma_{1, n}(\cdot ;$ $\left.\pi_{s}(x) ; I\right)$ из $\Sigma_{1, n}(I)$, хорошо приближающие эти многочлены.

Очевидно, что из леммы 6 следует оценка $\left\|\pi_{s}(\cdot ; x)\right\|_{L_{\infty}(I)} \leqslant(1+\tilde{c})\|x\|_{L_{\infty}(I)}$, где $\tilde{c}=\tilde{c}(s)$. Но тог да существуют сплайны $\sigma_{1, n}\left(\cdot ; \pi_{s}(x) ; I\right) \in \Sigma_{1, n}(I)$ такие, что

$$
\left\|\pi_{s}(\cdot)-\sigma_{1, n}\left(\cdot ; \pi_{s}(x) ; I\right)\right\|_{L_{\infty}(I)} \leqslant c n^{-1}, \quad n \geqslant 1
$$

где $c=c(s)$. Определяя в случае $r<s$ сплайны

$$
\sigma_{r, n}(t ; x ; I):=\sigma_{1, n}(t ; \tilde{x} ; I)+\sigma_{1, n}\left(t ; \pi_{s}(x) ; I\right), \quad t \in I,
$$

и учитьвая неравенства (4.2), приходим к оценке

$$
\left\|x(\cdot)-\sigma_{r, n}(\cdot ; x ; I)\right\|_{L_{\infty}(I)} \leqslant c n^{-1}, \quad n \geqslant 1, \quad r, s \in \mathbb{N}, \quad r<s,
$$

где $c=c(s)$. Но тогда из (4.4) и (4.5) следует, что

$$
E\left(\Delta_{+}^{s} B_{\infty}, \Sigma_{r, n}\right)_{L_{\infty}} \leqslant c n^{-1}, \quad n \geqslant 1, \quad r, s \in \mathbb{N}
$$

где $c=c(s)$.

В случае $s>1$ аналогичная (4.6) оценка верна и для наилучших приближений рациональными функциями. Действительно, полагая $\rho_{n}(t ; x) \equiv 0$ при $n<s / 2$, а при $n \geqslant s / 2$ полагая

$$
\rho_{2 n}(t):=\rho_{2 n}(t ; \tilde{x})+\pi_{s}(t ; x), \quad t \in I,
$$


где $\rho_{2 n}(\cdot ; \tilde{x})$ удовлетворяет неравенству $(4.3)$, приходим к оценке

$$
E\left(\Delta_{+}^{s} B_{\infty}, R_{n}\right)_{L_{\infty}} \leqslant c n^{-1}, \quad n \geqslant 1, s \in \mathbb{N}, \quad s>1,
$$

где $c=c(s)$.

Оценки же

$$
\begin{array}{ll}
E\left(\Delta_{+}^{s} B_{p}, \Sigma_{r, n}\right)_{L_{p}} \leqslant 1, & n \geqslant 1, \quad 1 \leqslant p<\infty, \quad r, s \in \mathbb{N}, \\
E\left(\Delta_{+}^{1} B_{\infty}, R_{n}\right)_{L_{\infty}} \leqslant 1, & n \geqslant 1,
\end{array}
$$

являются тривиальными.

Кроме того, из (4.6) и (4.8) в силу (4.42) следуют, соответственно, оценки

$$
d_{n}\left(\Delta_{+}^{s} B_{\infty}\right)_{L_{\infty}}^{p s d} \leqslant c n^{-1}, \quad n \geqslant 1, \quad s \in \mathbb{N}
$$

и

$$
d_{n}\left(\Delta_{+}^{s} B_{p}\right)_{L_{p}}^{p s d} \leqslant c, \quad n \geqslant 1, \quad 1 \leqslant p<\infty, \quad s \in \mathbb{N},
$$

где $c=c(s)$. Таким образом, все оценки сверху в теореме 1 установлены.

Объединяя при $s>1$ оценки снизу (3.9), (3.39) и (3.41) с оценками сверху (4.6), (4.7) и (4.11), получаем соотношение (1.1). При $s=1$ соотношение (1.2) следует из (3.9), $(3.39),(4.6)$ и (4.11), а соотношение (1.3) - из (3.43) и (4.9). Объединив оценки (3.36), $(3.40),(3.42),(4.8),(4.10)$ и (4.12), получаем соотношение (1.4). Теорема 1 доказана.

\section{СПИСОК ЦИТИРОВАННОЙ ЛИТЕРАТУРЫ}

[1] Bullen P.S. A criterion for $n$-convexity // Pacific J. Math. 1971. V. 36. P. 81-98.

[2] Roberts A. W., Varberg D. E. Convex Functions. New York: Academic Press, 1973.

[3] Pečarić J. E., Proschan F., Tong Y. L. Convex Functions, Partial Orderings, and Statistical Applications. Mathematics in Science and Engineering. V. 187. Boston: Academic Press, 1992.

[4] Буланов А. П. О порядке приближения выпуклых функций рациональными функциями // Изв. АН СССР. Сер. матем. 1969. Т. 33. № 5. С. 1132-1148.

[5] Попов В. А., Петрушев П. П. Точный порядок наилучшего равномерного приближения вьпуклых функций рациональными функциями // Матем. сб. 1977. Т. 103(145). №2(6). C. $285-292$.

[6] Haussler D. Decision theoretic generalizations of the PAC model for neural net and other learning applications // Information and Computation. 1992. V. 100. P. 78-150.

[7] Ratsaby J., Maiorov V. Generalization of the PAC-model for learning with partial information // Proc. of the 3rd European Conference on Computational Learning Theory (EuroCOLT 97). Berlin: Springer-Verlag, 1997.

[8] Ratsaby J., Maiorov V. On the value of partial information for learning from examples // J. Complexity. 1997. V. 13. P. 509-544.

[9] Lorentz G. G., van Golitschek M., Makovoz Y. Constructive Approximation, Advanced Problems. New York: Springer-Verlag, 1996.

[10] Maiorov V., Ratsaby J. The degree of approximation of sets in Euclidian space using sets with bounded Vapnik-Chervonenkis dimension // Discrete Appl. Math. 1998. V. 88. P. 81-93.

[11] Haussler D. Sphere packing numbers for subsets of the boolean $n$-cube with bounded Vapnik-Chervonenkis dimension // J. Combin. Theory. Ser. A. 1995. V. 69. P. 217-232.

[12] Maiorov V., Ratsaby J. On the degree of approximation by manifolds of finite pseudo-dimension // Constr. Approximation. 1999. V. 15. P. 291-300.

[13] Коновалов В. Н. Формосохраняющие поперечники типа Колмогорова классов $s$-монотонных интегрируемых функций // Укр. матем. ж. 2004. Т. 55. № 7. С. 901-926. 